Homology, Homotopy and Applications, vol.21(2), 2019, pp.83-106

\title{
REGIONS OF ATTRACTION, LIMITS AND END POINTS OF AN EXTERIOR DISCRETE SEMI-FLOW
}

\author{
J.M. GARCÍA CALCINES, L.J. HERNÁNDEZ PARICIO, \\ M. MARAÑÓN GRANDES AND M.T. RIVAS RODRÍGUEZ
}

(communicated by Graham Ellis)

\begin{abstract}
An exterior space is a topological space equipped with a distinguished quasi-filter of open subsets (closed by finite intersections) that we call externology. For an exterior space one can consider limits, bar-limits and different sets of end points (Steenrod, Čech, BrownGrossman).

In this work we analyze relations between exterior spaces and discrete semi-flows. In order to do this we introduce the notion of exterior discrete semi-flow, which is a mixture of exterior space and discrete semi-flow. We see that any classical discrete semi-flow can be provided with the structure of an exterior discrete semi-flow by taking the quasi-filter of right-absorbing open subsets. Such a family of open subsets is used to study the relations between limits and periodic points and connections between bar-limits and omega-limits. The different notions of end points are used to decompose the region of attraction of an exterior discrete semi-flow as a disjoint union of basins of end points. We also analyze the exterior discrete semi-flow structure induced by the family of open neighborhoods of a given sub-semi-flow.
\end{abstract}

\section{Introduction}

The homotopy theory of exterior spaces has connections with proper homotopy and (strong) shape theories as well as with the homotopy theory of pro-spaces and simplicial sets $[\mathbf{2 6}, \mathbf{2 7}, \mathbf{2 1}, \mathbf{5}]$. These theories are interrelated and some homotopy invariant groups are usually considered in order to distinguish spaces or to characterize some homotopy equivalences. For instance, Steenrod, Borsuk-Čech and Brown-Grossman homotopy groups can be given in all these contexts. Moreover, exterior spaces theory has proven to be very useful in the study of topological invariants in several settings such as proper homotopy theory and its numerical invariants or shape theory $([4,6,8,12,13,14,15])$. Also, some applications of exterior spaces have been

Partially supported by Ministerio de Economía y Competitividad (grant MTM2016-78647-P) and University of La Rioja (projects: APPI16/03, EGI16/42).

Received June 19, 2018; published on December 19, 2018.

2010 Mathematics Subject Classification: 54H20, 55P57, 55P55.

Key words and phrases: discrete semi-flow, exterior space, limit space, end point, end space, exterior discrete semi-flow.

Article available at http://dx.doi.org/10.4310/HHA.2019.v21.n2.a6

Copyright (C) 2018, International Press. Permission to copy for private use granted. 
developed to the study of continuous dynamical systems (flows) in $[16,17,18]$. The notion of absorbing open subset of a dynamical system (i.e., an open subset that contains the "future part" of all the trajectories) gives one of the key points to connect the seemingly different theories of dynamical systems and exterior spaces. Indeed, the family of all absorbing open subsets is a quasi-filter which gives the structure of an exterior space to the flow under study. The limit space and end space of this exterior space are used in $[\mathbf{1 6}, \mathbf{1 8}]$ to construct limit spaces and end spaces of a given flow. Such constructions enabled us to study relationships between exterior-theoretic notions of a continuous flow and the usual notions of omega-limits, first prolongational limits and several types of almost periodic points (Poisson-stable points, non-wandering points). We can also use the exterior end points (of Freudenthal type, see [10]) to obtain an induced decomposition of a dynamical system as a disjoint union of basins of end points. In [17], the theory of exterior spaces has also been used to construct a $\check{C}_{0}^{\mathrm{r}}$-completion and a $\check{C}_{0}^{\mathrm{l}}$-completion of a dynamical system. For any given flow $X$, two maps $X \rightarrow \check{C}_{0}^{\mathrm{r}}(X)$ and $X \rightarrow \check{C}_{0}^{\mathrm{l}}(X)$ are constructed and, when one of these maps is a homeomorphism, one has the class of $\check{C}_{0}^{\mathrm{r}}$-complete and $\check{C}_{0}^{1}$-complete flows, respectively. The advantage of working with a complete flow is that one has interesting relations between the topological properties (separability properties, compactness, convergence of nets, etc.) and dynamical properties (periodic points, omega limits, attractors, repellers, etc.). These results confirm the importance of the purely topological behavior of a continuous dynamical system in many, radically different in principle, situations (differential equations, non-linear analysis, transformation groups, etc.). As known, the topological techniques in dynamical systems were initially introduced by Poincaré $[24,25]$ and Birkhoff [3].

Taking into account the deep relations between exterior spaces and continuous dynamical systems, commented in the paragraph above, we have set as the main general objective of this paper to find out new links between exterior spaces and the dynamical properties of discrete dynamical systems. Discretization processes and the suspension of the Poincaré first return map and other constructions give a nice interdependence of the properties of discrete and continuous dynamical systems, see [9]. As a consequence, many of the properties, results and applications given in $[\mathbf{1 6}, \mathbf{1 7}, \mathbf{1 8}]$ must have a counterpart of notions and results that can be developed and proved for discrete dynamical systems. We may consider four important notions: continuous flows, continuous semi-flows, discrete flows and discrete semi-flows. It is worth mentioning some obvious differences between the properties of these structures: In a semi-flow, we are dealing with a semi-group of continuous maps instead of a group of homeomorphisms. This fact implies that for semi-flows the construction and properties of left omega-limits are quite different to those of right omega-limits. A continuous flow has the nice property that all the points in a trajectory are in the same path component; however, we cannot ensure such a nice property for discrete semi-flows. These differences have to be taken into account when one analyzes the interrelations between the theory of exterior spaces and the theory of discrete semi-flows. Subsequently, one has some similarities with the results and tools given in $[16,17,18]$, but new (non-analogous) techniques ought to be developed for a better analysis of discrete semi-flows. For instance, it is interesting to remark that, for continuous dynamical systems an analogue of the Borsuk-Čech invariant $\check{\pi}_{0}$ has played an important role in order to divide a continuous flow into a disjoint union of basins of end points given by 
the functor $\check{\pi}_{0}$. Nevertheless, for discrete semi-flows it is better to use the analogue of the Brown-Grossman invariant $\pi_{0}^{B G}$ instead of that of the functor $\check{\pi}_{0}$. The notion of Brown-Grossman end points is more natural for discrete semi-flows but this technique was not introduced in the previous applications of exterior spaces to continuous flows.

The techniques presented in this paper give a nice connection between notions associated to an exterior space and dynamic notions associated to a discrete semiflow. Indeed, for exterior discrete semi-flows we have the region of attraction of its externology as well as the limit and bar-limit of its externology. We also have different notions of end points (at least, one can consider three types of end points which are given by the analogues of the 0-dimensional homotopy invariants of types: BorsukČech $\check{\pi}_{0}$, Steenrod $\pi_{0}^{S}$ and Brown-Grossman groups $\pi_{0}^{B G}$ ). But, in discrete dynamic systems, one also has the classical notion of region of attraction of a right-invariant subset, the omega-limit of a point, periodic points, basins of $n$-cycles, etc. Then, we have discovered the existence of strong links between the regions of attraction of an externology and the regions of attraction of a right-invariant subset (see Theorem 5.6 and Theorem 5.7), between the notion of limit and the subset of periodic points (see Theorem 7.5), between the bar-limit and the notion of omega-limit (see Theorem 7.12), between the basin of an end point of Borsuk- Cech type and the basin of a fixed point and, finally, between the basin of an end point of Brown-Grossman type and the basin of a periodic point and of an $n$-cycle.

There are some other interesting interrelations of exterior spaces and discrete semiflows that have not been analyzed in this first approach of our present study. However, we think that the use of adequate quasi-filters of open subsets will also permit us to study questions relating to sensitivity to the initial conditions, stability problems and other dynamical questions connected to higher dimension homotopy groups of exterior spaces.

\section{Preliminaries}

\subsection{Exterior spaces}

In this subsection we recall some definitions and properties related to exterior spaces. For a deeper study of the category of exterior spaces we refer the reader to $[11,19]$.

Given a topological space $X$ with a topology $\mathbf{t}_{X}$ and a subset $A \subset X$, the closure of $A$ in $X$ is denoted by $\bar{A}$ and the interior by $\AA$ or $\operatorname{Int}(A)$.

Definition 2.1. Let $X$ be a topological space. An externology on $X$ is a non-empty collection $\varepsilon(X)$ of open subsets which is closed under finite intersections and such that, if $E \in \varepsilon(X)$ and $U$ is an open subset such that $E \subset U$, then $U \in \varepsilon(X)$. If an open subset is a member of $\varepsilon(X)$, then it is said to be an exterior open subset.

An exterior space $(X, \varepsilon(X))$ consists of a topological space $X$ together with an externology $\varepsilon(X)$. When it is clear from the context, we will shorten the notation by just writing $X$ instead of $(X, \varepsilon(X))$.

A map $f:(X, \varepsilon(X)) \rightarrow\left(X^{\prime}, \varepsilon\left(X^{\prime}\right)\right)$ is said to be an exterior map if it is continuous and $f^{-1}\left(E^{\prime}\right) \in \varepsilon(X)$, for all $E^{\prime} \in \varepsilon\left(X^{\prime}\right)$. 
For a topological space $X$ we can consider the co-compact externology

$$
\varepsilon^{\mathrm{c}}(X)=\{E \subset X \mid X \backslash E \text { is closed compact }\} .
$$

We denote $\mathbb{R}, \mathbb{R}_{+}$and $\mathbb{N}$ the exterior spaces determined by the usual topology and co-compact externology on the sets of real numbers $\mathbb{R}$, non-negative real numbers $\mathbb{R}_{+}$ and natural numbers $\mathbb{N}=\{0,1,2, \ldots\}$, respectively.

The category of exterior spaces and exterior maps is denoted by $\mathbf{E}$, and the category of topological spaces and continuous maps by Top.

An important role in this paper will be played by the following construction: Let $\left(X, \varepsilon(X), \mathbf{t}_{X}\right)$ be an exterior space, and let $\left(Y, \mathbf{t}_{Y}, \tau\right)$ be a set $Y$ endowed with two topologies $\mathbf{t}_{Y}, \tau$ such that $\mathbf{t}_{Y} \subset \tau$. For $y \in Y$ we denote by $\tau_{y}$ the family of open neighborhoods of $(Y, \tau)$ at $y$. Given the topological spaces $\left(X, \mathbf{t}_{X}\right),\left(Y, \mathbf{t}_{Y}\right)$, we consider on $X \times Y$ the product topology which is denoted by $\mathbf{t}_{X \times Y}$ and the externology $\varepsilon\left(X \overline{\times}_{\tau} Y\right)$ given by those $E \in \mathbf{t}_{X \times Y}$ such that for each $y \in Y$ there exists $U_{y} \in \tau_{y}$ and $T^{y} \in \varepsilon(X)$ such that $T^{y} \times U_{y} \subset E$. The resulting exterior space $\left(X \times Y, \varepsilon\left(X \overline{\times}_{\tau} Y\right), \mathbf{t}_{X \times Y}\right)$ will be denoted by $X \overline{\times}_{\tau} Y$.

In some contexts, for instance working with exterior homotopies, it is frequent to take $\tau=\mathbf{t}_{Y}$. For these cases, we will use the shorter notation

$$
X \overline{\times} Y=\left(X \times Y, \varepsilon\left(X \overline{\times}_{\mathbf{t}_{Y}} Y\right), \mathbf{t}_{X \times Y}\right)=X \overline{\times}_{\mathbf{t}_{Y}} Y .
$$

Therefore, for these cases we have an induced functor:

$$
(\cdot) \overline{\times}(\cdot): \mathbf{E} \times \mathbf{T o p} \rightarrow \mathbf{E}, \quad(X, Y) \mapsto X \overline{\times} Y .
$$

When $Y$ is a compact space, we have that $E$ is an exterior open subset of $X \overline{\times} Y$ if and only if it is an open subset and there exists $G \in \varepsilon(X)$ such that $G \times Y \subset E$. Furthermore, if $Y$ is a compact space and $\varepsilon(X)=\varepsilon^{\mathrm{c}}(X)$, then $\varepsilon(X \overline{\times} Y)$ coincides with $\varepsilon^{\mathrm{c}}(X \times Y)$ the externology of the complements of closed-compact subsets of $X \times Y$. We also note that if $Y$ is a discrete space, then $E$ is an exterior open subset of $X \overline{\times} Y$ if and only if it is open and for each $y \in Y$ there is $T^{y} \in \varepsilon(X)$ such that $T^{y} \times\{y\} \subset E$.

Given $f, g: X \rightarrow Y$ in $\mathbf{E}$, it is said that $f$ is exterior homotopic to $g$ if there is an exterior homotopy $H: X \overline{\times} I \rightarrow Y$ from $f$ to $g$. Denote by $\pi \mathbf{E}$ and $\pi$ Top the exterior homotopy category and the usual homotopy category corresponding to $\mathbf{E}$ and Top, respectively. Given $X, Y$ two exterior spaces, the set of exterior homotopy classes from $X$ to $Y$ will be denoted by $\pi \mathbf{E}(X, Y)$. Its elements $[f]$ are homotopy equivalence classes of exterior maps $f: X \rightarrow Y$. Similar notation is used in the case of topological spaces.

We recall that, for a topological space $Y, \pi_{0}(Y)$ denotes the set of path components of $Y$ and we have a quotient map $Y \rightarrow \pi_{0}(Y)$. Note that a continuous map $f: Y \rightarrow Y^{\prime}$ induces a natural map $\pi_{0}(f): \pi_{0}(Y) \rightarrow \pi_{0}\left(Y^{\prime}\right)$.

An inverse system of sets (or topological spaces) is a functor $Z: I \rightarrow$ Sets, where $I$ is a directed set and Sets is the category of sets (resp., considering Top). The functor $Z$ carries $i \geqslant j, i, j \in I$, to $Z_{j}^{i}: Z_{i} \rightarrow Z_{j}$. The inverse limit of $Z=\left\{Z_{i}\right\}$ is denoted by $\lim _{i \in I} Z_{i}$ (or just by $\lim Z_{i}$ ). An element of the inverse limit can be represented by an element $\left(z_{i}\right)_{i \in I}$ of the product $\prod_{i \in I} Z_{i}$ satisfying that $Z_{j}^{i}\left(z_{i}\right)=z_{j}, i \geqslant j$. We also have that if $\left\{Y_{i}\right\}$ is an inverse system of topological spaces, then $\left\{\pi_{0}\left(Y_{i}\right)\right\}$ is an inverse system of sets and one can consider the inverse $\operatorname{limit} \lim _{i \in I} \pi_{0}\left(Y_{i}\right)$. An element of $\lim _{i \in I} \pi_{0}\left(Y_{i}\right)$ is given by $\left(C_{i}\right)_{i \in I}$, where $C_{i}$ is a path component of $Y_{i}$ such 
that $Y_{j}^{i}\left(C_{i}\right) \subset C_{j}$ for $i \geqslant j$. For more results and properties about inverse systems, we refer the reader to [5].

Definition 2.2. Given an exterior space $X$, its externology $\varepsilon(X)$ can be considered as an inverse system of topological spaces, and we have the following notions:

The topological subspace

$$
L(X)=\lim _{E \in \varepsilon(X)} E=\bigcap_{E \in \varepsilon(X)} E
$$

will be called the limit space of $X$ and

$$
\bar{L}(X)=\lim _{E \in \varepsilon(X)} \bar{E}=\bigcap_{E \in \varepsilon(X)} \bar{E}
$$

will be called the bar-limit space of $X$.

The $\check{\pi}_{0}$-end set of $X$ is given by

$$
\check{\pi}_{0}(X)=\lim _{E \in \varepsilon(X)} \pi_{0}(E) .
$$

The $\check{\bar{\pi}}_{0}$-end set of $X$ is given by

$$
\check{\pi}_{0}(X)=\lim _{E \in \varepsilon(X)} \pi_{0}(\bar{E}) .
$$

The $\pi_{0}^{\mathrm{BG}}$-end set of $X$ is given by

$$
\pi_{0}^{\mathrm{BG}}(X)=\pi \mathbf{E}(\mathbb{N}, X) .
$$

The $\pi_{0}^{\mathrm{S}}$-end set of $X$ is given by

$$
\pi_{0}^{\mathrm{S}}(X)=\pi \mathbf{E}\left(\mathbb{R}_{+}, X\right) .
$$

\subsection{Discrete semi-flows}

Next, we recall some basic notions about discrete semi-flows. These notions can be given for a set or for a topological space.

Definition 2.3. A discrete semi-flow on a (topological space) set $X$ is a (continuous) map $\phi: \mathbb{N} \times X \rightarrow X$ such that:

(i) $\phi(0, x)=x, \forall x \in X$;

(ii) $\phi(n, \phi(m, x))=\phi(n+m, x), \forall x \in X, \forall n, m \in \mathbb{N}$.

A discrete semi-flow on $X$ will be denoted by $(X, \phi)$ and, when no confusion is possible, we will use $X$ and $n \cdot x=\phi(n, x)$ for short. Similarly, for a subset $S \subset \mathbb{N}$ we will denote $S \cdot x=\{n \cdot x \mid n \in S\}$.

Given two discrete semi-flows $(X, \phi)$ and $(Y, \psi)$, a discrete semi-flow morphism $f:(X, \phi) \rightarrow(Y, \psi)$ is a (continuous) map $f: X \rightarrow Y$ such that $f(n \cdot x)=n \cdot f(x)$, for every $(n, x) \in \mathbb{N} \times X$. The category of discrete semi-flows (defined on topological spaces) will be denoted by $\mathbf{F}(\mathbb{N})$.

Remark 2.4. Note that giving a discrete semi-flow $(X, \phi)$ on a (topological space) set $X$ is equivalent to giving a (continuous) map $f=\phi^{1}: X \rightarrow X$. 
Given a discrete semi-flow $\phi: \mathbb{N} \times X \rightarrow X, n_{0} \in \mathbb{N}, x_{0} \in X$, we have the induced maps $\phi^{n_{0}}: X \rightarrow X, \phi^{n_{0}}(x)=\phi\left(n_{0}, x\right)$ and $\phi_{x_{0}}: \mathbb{N} \rightarrow X, \phi_{x_{0}}(n)=\phi\left(n, x_{0}\right)$.

For a discrete semi-flow $(X, \phi)$, a subset $A \subset X$ is said to be right invariant if $\phi^{1}(A) \subset A$ and it is said to be left invariant if $\left(\phi^{1}\right)^{-1}(A) \subset A$. A subset which is left-invariant and right-invariant it is said to be completely invariant.

Given two points $x, y \in X$, we have the following equivalence relation: $x \sim y$ if there are $k, l \in \mathbb{N}$ such that $\phi^{k}(x)=\phi^{l}(y)$. If $[x]$ is the equivalence class of $x$, note that $[x]$ is a completely invariant subset. Denote by $X / \sim$ the quotient set which has a trivial induced action. The subset $[x]$ of $X$ is the big orbit of $x$ and the subset $\mathbb{N} \cdot x$ is the trajectory of $x$.

Definition 2.5. Let $X$ be a discrete semi-flow and $x$ a point of $X$.

(i) $x$ is a fixed point if, for every $n \in \mathbb{N}, n \cdot x=x$.

(ii) $x$ is a periodic or cyclic point if there is $n \in \mathbb{N}, n \neq 0$, such that $n \cdot x=x$.

(iii) $x$ is an $m$-periodic point if $m \cdot x=x$.

(iv) $x$ is an $m$-cyclic point if $m \cdot x=x$ and if for $0<k<m$, then $k \cdot x \neq x$.

The right-invariant subsets of fixed, periodic, $m$-periodic and $m$-cyclic points of $X$ are denoted by $\operatorname{Fix}(X), P(X), P_{m}(X)$ and $C_{m}(X)$, respectively. From the definition, it is clear that $C_{m}(X) \subset P_{m}(X)$.

A net of a topological space $X$ is denoted by $x_{i}$, where we suppose that $i$ describes a directed set. In this paper, $[n,+\infty)$ denotes the subset $\{m \in \mathbb{N} \mid m \geqslant n\}$ and similarly $(n,+\infty)=\{m \in \mathbb{N} \mid m>n\}$. The following notions are given for topological spaces with a given semi-flow structure:

Definition 2.6. For a discrete semi-flow $(X, \phi)$, the omega-limit set of a point $x \in X$ (or right-limit set, or positive limit set) is given as follows:

$\Lambda(x)=\left\{y \in X \mid\right.$ there exists a net $n_{i} \rightarrow+\infty, n_{i} \in \mathbb{N}$, such that $\left.n_{i} \cdot x \rightarrow y\right\}$.

We note that the subset $\Lambda(x)$ admits the alternative definition

$$
\Lambda(x)=\bigcap_{n \geqslant 0} \overline{[n,+\infty) \cdot x}
$$

For a given subspace $S \subset X$, the set $\Lambda(S)=\bigcup_{x \in S} \Lambda(x)$ is called the omega-limit set of $S$.

A point $x \in X$ is said to be Poisson stable (or positively Poisson stable) if $x \in$ $\Lambda(x)$. We will denote by Poisson $(X)$ the right-invariant subset of Poisson stable points of $X$.

Note that $P(X) \subset$ Poisson $(X) \subset \Lambda(X)$. It is also easy to check that $\Lambda(x)$ and $\Lambda(X)$ are right-invariant subsets of $X$ and one also has that, for $x \in X, x$ and $\phi^{1}(x)$ have the same omega-limit.

Lemma 2.7. Let $(X, \phi)$ be a discrete semi-flow and let $S \subset X$. Then:

(i) $\phi^{1}(\Lambda(x)) \subset \Lambda(x)$,

(ii) $\phi^{1}(\Lambda(S)) \subset \Lambda(S)$,

(iii) $\Lambda\left(\phi^{1}(x)\right)=\Lambda(x)$. 
Remark 2.8. Observe that, if $X$ satisfies the first axiom of countability (for instance, when $X$ is metrizable), then we can consider sequences instead of nets in Definition 2.6.

Definition 2.9. Let $(X, \phi)$ be a discrete semi-flow and $x \in X$. It is said that $X$ is Lagrange stable at $x$ (or positively Lagrange stable at $x$ ) if $\overline{\mathbb{N} \cdot x}$ is a compact subset. $X$ is Lagrange stable if, for every $x \in X, X$ is Lagrange stable at $x$.

Definition 2.10. Let $(X, \phi)$ be a discrete semi-flow and let $S \subset X$.

The region of pseudo-attraction of $S$ is defined by

$$
\operatorname{PA}(S)=\{x \in X \mid \Lambda(x) \subset S\} .
$$

The region of weak-attraction of $S$ is defined by

$$
\mathrm{WA}(S)=\{x \in X \mid \Lambda(x) \cap S \neq \emptyset\} .
$$

The region of attraction of $S$ is defined by

$$
\mathrm{A}(S)=\{x \in X \mid \emptyset \neq \Lambda(x) \subset S\} .
$$

Lemma 2.11. Let $(X, \phi)$ be a discrete semi-flow and let $S \subset X$. Then:

(i) $\mathrm{PA}(S)=\mathrm{PA}(\emptyset) \cup \mathrm{A}(S), \emptyset=\mathrm{PA}(\emptyset) \cap \mathrm{A}(S)$;

(ii) $\mathrm{A}(S)=\mathrm{PA}(S) \cap \mathrm{WA}(S)$;

(iii) $\mathrm{PA}(S), \mathrm{WA}(S), \mathrm{A}(S)$ are completely invariant;

(iv) If $X$ is Lagrange stable at every point of $\mathrm{PA}(S)$, then

$$
\operatorname{PA}(\emptyset)=\emptyset \quad \text { and } \quad \operatorname{PA}(S)=\mathrm{A}(S) .
$$

Proof. (i) and (ii) follow from the definition and (iii) from the identity $\Lambda(x)=$ $\Lambda\left(\phi^{1}(x)\right)$, for every $x \in X$. (iv) follows from the Lagrange stability of $X$ and the fact that $\Lambda(x)$ is a non-empty compact subset of $X$, for any $x \in X$.

\section{Natural transformations for limit and end spaces of exterior spaces}

In subsection 2.1, for an exterior space, the notions of limit, bar-limit and different sets of end points were introduced. Now, we analyze some relationships between them.

Given an exterior space $(X, \varepsilon(X))$, the canonical maps

$$
E \subset \bar{E}, \quad E \rightarrow \pi_{0}(E), \quad \bar{E} \rightarrow \pi_{0}(\bar{E})
$$

induce a commutative diagram

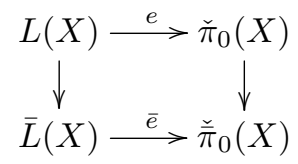

We can consider the shift map $s: \mathbb{N} \rightarrow \mathbb{N}$, given by $s(i)=i+1, i \in \mathbb{N}$. This exterior map induces the canonical map $S: \pi_{0}^{\mathrm{BG}}(X) \rightarrow \pi_{0}^{\mathrm{BG}}(X), S([\alpha])=[\alpha s]$, for any given exterior map $\alpha: \mathbb{N} \rightarrow X$. 
The inclusion map in : $\mathbb{N} \rightarrow \mathbb{R}_{+}$induces a natural transformation

$$
R_{X}: \pi_{0}^{\mathrm{S}}(X) \rightarrow \pi_{0}^{\mathrm{BG}}(X),
$$

given by $R_{X}([\alpha])=\left[\left.\alpha\right|_{\mathbb{N}}\right]$ for an exterior map $\alpha: \mathbb{R}_{+} \rightarrow X$. Then we can consider the diagram

$$
\pi_{0}^{\mathrm{S}}(X) \stackrel{R_{X}}{\longrightarrow} \pi_{0}^{\mathrm{BG}}(X) \stackrel{\mathrm{Id}}{\underset{S}{\longrightarrow}} \pi_{0}^{\mathrm{BG}}(X),
$$

where the image of $R_{X}$ is the equalizer of the identity and shift maps (see [19]).

Remark 3.1. In fact, there is a very interesting exact sequence of homotopy groups associated to an exterior space $X$ with a base ray $\alpha: \mathbb{R}_{+} \rightarrow X$ (i.e., $\alpha$ is an exterior map)

$$
\cdots \longrightarrow \pi_{q}^{\mathrm{S}}(X) \longrightarrow \pi_{q}^{\mathrm{BG}}(X) \stackrel{\mathrm{Id}-S}{\longrightarrow} \pi_{q}^{\mathrm{BG}}(X) \longrightarrow \pi_{q-1}^{\mathrm{S}}(X) \longrightarrow \cdots
$$

ending at dimension zero in the above diagram. These higher exterior homotopy invariants are powerful tools for the study and classification of exterior spaces, see $[5,8,19]$. In the next sections we will only consider the zero dimensional part of this sequence for the study of end points (and their basins) of an exterior discrete semi-flow. Similar exact sequences in the context of shape theory or pro-spaces can be seen in $[26,20]$.

The connection between the set of $\pi_{0}^{\mathrm{S}}$-end points and the set of $\check{\pi}_{0}$-end points of an exterior space is given by the natural transformation

$$
\eta_{X}: \pi_{0}^{\mathrm{S}}(X) \rightarrow \check{\pi}_{0}(X),
$$

where $\eta_{X}$ is defined by $\eta_{X}([\alpha])=\check{\pi}_{0}(\alpha)(+\infty)$ (observe that $\check{\pi}_{0}\left(\mathbb{R}_{+}\right)=\{+\infty\}$ ).

There is also a relationship between the sets of $\check{\pi}_{0}$-end points and $\pi_{0}^{\mathrm{BG}}$-end points of $X$, but we need exterior spaces with some additional conditions.

Definition 3.2. An exterior space $(X, \varepsilon(X))$ is said to be first-countable at infinity if $\varepsilon(X)$ contains a countable base $E_{0} \supset E_{1} \supset E_{2} \supset \cdots$; that is, each $E_{i} \in \varepsilon(X)$ and, for every $E \in \varepsilon(X)$, there is $i \in \mathbb{N}$ such that $E_{i} \subset E$.

Proposition 3.3. Let $(X, \varepsilon(X))$ be a first-countable at infinity exterior space. Then there is a canonical injective map $\theta_{X}: \check{\pi}_{0}(X) \rightarrow \pi_{0}^{\mathrm{BG}}(X)$ such that the image of this map is the set $\left\{a \in \pi_{0}^{\mathrm{BG}}(X) \mid S(a)=a\right\}$. Moreover, $\eta_{X}$ is surjective and the map $R_{X}$ factorizes as

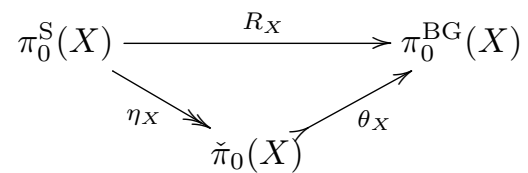

Consequently, for exterior spaces which are first-countable at infinity, $\theta_{X}: \check{\pi}_{0}(X) \rightarrow$ $\pi_{0}^{\mathrm{BG}}(X)$ is the equalizer of the identity and the shift map.

Proof. Since $X$ is first-countable at infinity, we have a countable base: $E_{0} \supset E_{1} \supset$ $E_{2} \supset \cdots$. By the definition of $\check{\pi}_{0}(X)$, it follows that $\check{\pi}_{0}(X)=\lim \pi_{0}\left(E_{i}\right)$. Therefore 
an end point $a \in \check{\pi}_{0}(X)$ can be represented by a sequence of path components $C_{0} \supset$ $C_{1} \supset C_{2} \supset \cdots$. For every $i \in \mathbb{N}$, take a point $x_{i} \in C_{i}$ and define a map $\alpha: \mathbb{N} \rightarrow X$ by $\alpha(i)=x_{i}$. Then the map $\theta_{X}: \check{\pi}_{0}(X) \rightarrow \pi_{0}^{\mathrm{BG}}(X)$ is given by $\theta_{X}(a)=[\alpha]$. It is easy to check that the definition of $\theta_{X}$ does not depend on the chosen base nor the chosen points and that it is injective.

Now if $x_{i}, x_{i+1} \in C_{i}$, then there is path $F_{i}: I \rightarrow C_{i}$ from $x_{i}$ to $x_{i+1}$. The exterior homotopy $F: \mathbb{N} \bar{x} I \rightarrow X$ given by $F(i, t)=F_{i}(t)$ satisfies that $F(i, 0)=x_{i}$ and $F(i, 1)=x_{i+1}$. This implies that $S\left(\theta_{X}(a)\right)=\theta_{X}(a)$. Conversely, take $\alpha: \mathbb{N} \rightarrow X$ and suppose that $S([\alpha])=[\alpha s]=[\alpha]$. Then there is an exterior homotopy $F: \mathbb{N} \overline{\times} I \rightarrow X$ from $\alpha$ to $\alpha s$ and we can define an exterior map $\beta: \mathbb{R}_{+} \rightarrow X$ by $\beta(r)=F(E(r)$, $r-E(r))$, where $E(r)$ is the integer part of $r \geqslant 0$. It is easy to check that $\theta_{X}\left(\check{\pi}_{0}(\beta)(+\infty)\right)=[\alpha]$. The rest of the proof follows in a straightforward way.

Remark 3.4. In order to obtain a more complete description of the relations between constructions and invariants associated to an exterior space and distinguished subsets of discrete semi-flows, the authors think that some further work needs to be done by using the following new notions: Given two exterior spaces $(X, \varepsilon(X)),\left(X^{\prime}, \varepsilon\left(X^{\prime}\right)\right)$ a bar-exterior map $f:(X, \varepsilon(X)) \rightarrow\left(X^{\prime}, \varepsilon\left(X^{\prime}\right)\right)$ is a continuous map $f: X \rightarrow X^{\prime}$ such that for every $E^{\prime} \in \varepsilon\left(X^{\prime}\right)$, there is $E \in \varepsilon(X)$ such that $f(\bar{E}) \subset \overline{E^{\prime}}$. Note that an exterior map is always a bar-exterior map. This yields to a large category $\overline{\mathbf{E}}$, a new homotopy category $\pi(\overline{\mathbf{E}})$ and new invariants:

1. The $\bar{\pi}_{0}^{\mathrm{BG}}$-end set of $X$ is given by $\bar{\pi}_{0}^{\mathrm{BG}}(X)=\pi(\overline{\mathbf{E}})(\mathbb{N}, X)$.

2. The $\bar{\pi}_{0}^{\mathrm{S}}$-end set of $X$ is given by $\bar{\pi}_{0}^{\mathrm{S}}(X)=\pi(\overline{\mathbf{E}})\left(\mathbb{R}_{+}, X\right)$.

It is interesting to observe that taking the categories $\overline{\mathbf{E}}$ and $\pi(\overline{\mathbf{E}})$, there is a new exact sequence of homotopy groups associated to an exterior space $X$ with a base ray $\alpha: \mathbb{R}_{+} \rightarrow X$

$$
\cdots \longrightarrow \bar{\pi}_{q}^{\mathrm{S}}(X) \longrightarrow \bar{\pi}_{q}^{\mathrm{BG}}(X) \stackrel{\mathrm{Id}-S}{\longrightarrow} \bar{\pi}_{q}^{\mathrm{BG}}(X) \longrightarrow \bar{\pi}_{q-1}^{\mathrm{S}}(X) \longrightarrow \cdots .
$$

The new "bar-invariants" can be related to invariants above using the exact sequences associated to the pairs $(\bar{E}, E)_{E \in \varepsilon(X)}$ that will reflect the differences between the two types of invariants.

In the present paper, we add some remarks about some possible new results connected with the notion of bar-exterior map. The new notions are related to pure topological versions of Julia and Fatou sets and a more complete study needs to be done to clarify these interesting connections.

\section{Exterior discrete semi-flows}

In this section we introduce the notion of exterior discrete semi-flow, which combines the notions of exterior space and discrete semi-flow.

Definition 4.1. Let $X$ be an exterior space. An exterior discrete semi-flow is a discrete semi-flow $\phi: \mathbb{N} \times X \rightarrow X$ such that, for any $n \in \mathbb{N}, \phi^{n}: X \rightarrow X$ is exterior (this is equivalent to the simpler condition $\phi^{1}: X \rightarrow X$ is exterior).

An exterior discrete semi-flow $X$ is said to be $\mathbf{d}$-exterior if it satisfies that $\phi_{x}: \mathbb{N} \rightarrow$ $X$ is exterior, for every $x \in X$. 
An exterior discrete semi-flow morphism of exterior discrete semi-flows $f: X \rightarrow Y$ is a discrete semi-flow morphism such that $f$ is exterior.

Remark 4.2. The condition which asserts that $\phi_{x}: \mathbb{N} \rightarrow X$ is exterior for every $x \in X$ can be replaced by the requirement that $\phi: \mathbb{N}_{\mathbf{x}} X \rightarrow X$ is exterior, where $\mathbf{d}$ stands for the discrete topology on $X$ (remember the general construction $X \overline{\times}_{\tau} Y$ given at the preliminaries). Observe that this second topology $\mathbf{d}$ considered on $X$ is just used in order to give the corresponding structure of exterior space on $\mathbb{N} \bar{x}_{\mathbf{d}} X$. This is the reason for choosing the terminology "d-exterior" in the definition above.

Denote by $\mathbf{E F}(\mathbb{N})$ the category of exterior discrete semi-flows and by $\mathbf{E}^{\mathbf{d}} \mathbf{F}(\mathbb{N})$, the full subcategory of $\mathbf{d}$-exterior discrete semi-flows.

We adopt the following notational convention: an exterior discrete semi-flow will be denoted by a triplet $(X, \phi, \varepsilon(X))$. Nevertheless, sometimes when the action $\phi$ or the externology are clear in a particular context, we will shorten the notation and we will use $(X, \varepsilon(X))$ or $(X, \phi)$; moreover, in many cases the notation will be reduced to $X$.

We have defined above the limit space of an exterior space. In particular, since an exterior discrete semi-flow $X$ is an exterior space, we can consider the limit space $L(X)$ and the bar-limit space $\bar{L}(X)$.

Proposition 4.3. Let $(X, \phi, \varepsilon(X))$ be an exterior discrete semi-flow. Then $L(X)$ and $\bar{L}(X)$ are right-invariant.

Proof. Denote $f=\phi^{1}$. Since $f$ is an exterior map, it follows that $f^{-1}(E) \in \varepsilon(X)$ for every $E \in \varepsilon(X)$. Then one has that

$$
f^{-1}(L(X))=f^{-1}\left(\bigcap_{E \in \varepsilon(X)} E\right)=\bigcap_{E \in \varepsilon(X)} f^{-1}(E) \supset \bigcap_{E \in \varepsilon(X)} E=L(X) .
$$

This implies that $f(L(X)) \subset L(X)$. For $\bar{L}(X)$, the proof is similar using also the fact that if $f$ is continuous, $f(\bar{E}) \subset \overline{f(E)}$ for every $E \in \varepsilon(X)$.

For an exterior discrete semi-flow $X$, one has that the exterior map $\phi^{1}: X \rightarrow X$ induce the maps $\pi_{0}^{S}\left(\phi^{1}\right), \pi_{0}^{\mathrm{BG}}\left(\phi^{1}\right), \check{\pi}_{0}\left(\phi^{1}\right), \check{\pi}_{0}\left(\phi^{1}\right)$ that give canonical discrete semiflow structures on the corresponding sets $\pi_{0}^{S}(X), \pi_{0}^{\mathrm{BG}}(X), \check{\pi}_{0}(X), \check{\pi}_{0}(X)$. Therefore, the exterior homotopy invariants $\pi_{0}^{S}(X), \pi_{0}^{\mathrm{BG}}(X), \check{\pi}_{0}(X), \check{\bar{\pi}}_{0}(X)$ can be enriched with a discrete semi-flow structure.

\section{The regions of attraction of an exterior discrete semi-flow}

Now, suppose that $(X, \phi, \varepsilon(X))$ is an exterior discrete semi-flow. Consider

$$
D(X)=\left\{x \in X \mid \phi_{x} \text { is exterior }\right\} .
$$

In a similar way, one can define $\bar{D}(X)$ as the set of points $x \in X$ such that, for every $E \in \varepsilon(X)$, there is $n_{E} \in \mathbb{N}$ such that, for every $n \geqslant n_{E}, \phi^{n}(x) \in \bar{E}$. The subspace $\bar{D}(X)$ has many similar properties to those of the subspace $D(X)$, whose proofs are left to the reader. However, the most difficult proofs relating to $\bar{D}(X)$ will be included in this paper. 
Proposition 5.1. For an exterior discrete semi-flow $X=(X, \phi, \varepsilon(X))$, the subspace $D(X)$ is completely invariant and, with the relative externology (i.e., the family of intersections of the form $D(X) \cap E, E \in \varepsilon(X))$, is a d-exterior discrete semi-flow.

Proof. Denote $D=D(X)$. Observe that $\phi_{\phi^{1}(x)}=\phi_{x} s$, where the shift $s, s(n)=$ $n+1$, is an exterior map. This implies that, if $x \in D$, then $\phi^{1}(x) \in D$.

Now, in order to prove that $D$ is left-invariant, suppose that $\phi^{1}(x)=y$ and $y \in D$. Given an exterior open subset $E$, there is $n \geqslant 1$ such that $\phi^{m}(y) \in E$, for every $m \geqslant n$. Then $\phi^{k}(x) \in E$, for every $k \geqslant n+1$. This implies that $x \in D$.

Finally, since $X$ is an exterior discrete semi-flow, taking on $D$ the relative externology, it follows that $\phi^{n}: D \rightarrow D$ is exterior, for every $n \in \mathbb{N}$. By the definition of $D$ one has that $\phi_{x}: D \rightarrow D$ is exterior, for every $x \in D$. Therefore, $D$ with the relative externology is a $\mathbf{d}$-exterior discrete semi-flow.

Definition 5.2. Suppose that $X=(X, \phi, \varepsilon(X))$ is an exterior discrete semi-flow. The d-exterior discrete semi-flow $D(X)$ is said to be the region of pseudo-attraction of the externology $\varepsilon(X)$. The exterior discrete semi-flow $\bar{D}(X)$ is said to be the region of pseudo-bar-attraction of the externology $\varepsilon(X)$.

Sometimes, if $X$ is an exterior discrete semi-flow, we say that $D(X)$ is the region of pseudo-attraction of $X$ (instead of $\varepsilon(X))$ and similarly for $\bar{D}(X)$.

Remark 5.3. Notice that the canonical functor $\mathbf{E F}(\mathbb{N}) \rightarrow \mathbf{E}^{\mathbf{d}} \mathbf{F}(\mathbb{N})$, which carries $X$ to $D(X)$, is right adjoint to the inclusion functor $\mathbf{E}^{\mathbf{d}} \mathbf{F}(\mathbb{N}) \rightarrow \mathbf{E F}(\mathbb{N})$.

Remark 5.4. Using the notion of bar-exterior map mentioned in Remark 3.4 one has the following alternative definition of $\bar{D}(X)$ for an exterior discrete semi-flow $X$, $\bar{D}(X)=\left\{x \in X \mid \phi_{x}\right.$ is bar-exterior $\}$.

If $X=(X, \phi, \varepsilon(X))$ is an exterior discrete semi-flow, the inclusion $D(X) \subset X$ of exterior spaces induces the transformation $L(D(X)) \rightarrow L(X)$.

Proposition 5.5. If $X=(X, \phi, \varepsilon(X))$ is an exterior discrete semi-flow, then $L(D(X))=L(X)$ (similarly, $\bar{L}(\bar{D}(X))=\bar{L}(X))$.

For an exterior discrete semi-flow, some relations between regions of attraction associated to its externology and standard regions of attraction as a discrete semiflow as the following:

Theorem 5.6. Suppose that $X=(X, \phi, \varepsilon(X))$ is an exterior discrete semi-flow. Then:

(i) $D(X) \subset \bar{D}(X) \subset \operatorname{PA}(\bar{L}(X))$;

(ii) If $(X, \phi)$ is Lagrange stable at every point in $\bar{D}(X)$, then $\bar{D}(X) \subset \mathrm{A}(\bar{L}(X))$;

(iii) If $(X, \phi)$ is Lagrange stable at every point in $\mathrm{A}(L(X))$, then $\mathrm{A}(L(X)) \subset D(X)$;

(iv) If $(X, \phi)$ is Lagrange stable at every point in $\mathrm{PA}(L(X))$, then $\mathrm{PA}(L(X)) \subset$ $D(X)$

(v) If $(X, \phi)$ is Lagrange stable at every point in $\operatorname{PA}(L(X))$ and $L(X)=\bar{L}(X)$, then $D(X)=\operatorname{PA}(L(X))=A(L(X))$.

Proof.

(i) Obviously, $D(X) \subset \bar{D}(X)$. If $x \in \bar{D}(X)$ and $E \in \varepsilon(X)$, there is $n_{E} \in \mathbb{N}$, such that $\left[n_{E}, \infty\right) \cdot x \subset \bar{E}$. Then $\overline{[n, \infty) \cdot x} \subset \bar{E}$. This implies that $\Lambda(x) \subset \bar{E}$, for every $E \in \varepsilon(X)$. Hence, $\Lambda(x) \subset \bar{L}(X)$. 
(ii) If $(X, \phi)$ is Lagrange stable at a point $x$, we also have that $\Lambda(x) \neq \emptyset$.

(iii) Suppose that $x \in A(L(X))$ and $\phi_{x}$ is not exterior. Then there is $E \in \varepsilon(X)$ and an increasing sequence $n_{1}<n_{2}<\cdots$ such that $\phi^{n_{k}}(x) \in X \backslash E$. Since $X$ is Lagrange stable at $x$, there is a sub-net $n_{k_{i}}$ such that $n_{k_{i}} \cdot x \rightarrow y \in X \backslash E$. But we also have that $y \in \Lambda(x) \backslash L(X)$ and this is a contradiction.

(iv) This can be proved in a similar way.

(v) This is a consequence of previous results and Lemma 2.11(iv).

Theorem 5.7. Suppose that $X=(X, \phi, \varepsilon(X))$ is an exterior discrete semi-flow and $(X, \phi)$ is Lagrange stable at every point in $\mathrm{PA}(\bar{L}(X))$. Then:

(i) $\operatorname{PA}(\bar{L}(X)) \backslash \mathrm{WA}(\bar{L}(X) \backslash L(X)) \subset \bar{D}(X)$,

(ii) If, in addition, $\mathrm{WA}(\bar{L}(X) \backslash L(X))=\emptyset$, one has that

$$
\mathrm{A}(\bar{L}(X))=\bar{D}(X) .
$$

Proof.

(i) Suppose that $x \in \operatorname{PA}(\bar{L}(X)) \backslash \mathrm{WA}(\bar{L}(X) \backslash L(X))$ and $x \notin \bar{D}(X)$. Then there is $E \in \varepsilon(X)$ and an increasing sequence $n_{1}<n_{2}<\cdots$ such that $\phi^{n_{k}}(x) \in X \backslash \bar{E}$. Since $X$ is Lagrange stable at $x$, there is a sub-net $n_{k_{i}}$ such that $n_{k_{i}} \cdot x \rightarrow y \in$ $X \backslash E$. Then $y \in \Lambda(x) \backslash L(X)$ and $x \in \mathrm{WA}(\bar{L}(X) \backslash L(X))$, which is a contradiction.

(ii) It follows by (i), Theorem 5.6(i) and Lemma 2.11.

\section{Basins associated to end points in a region of attraction}

We note that, for an exterior discrete semi-flow $X=(X, \phi, \varepsilon(X))$, each trajectory of a point of $D(X)$ has an end point given as follows:

If $x \in D(X)$, we have the exterior map $\phi_{x}: \mathbb{N} \rightarrow X, \phi_{x}(n)=\phi(n, x)$, which determines an end point $\left[\phi_{x}\right] \in \pi_{0}^{\mathrm{BG}}(X)$. Then the following canonical map is obtained

$$
\omega: D(X) \rightarrow \pi_{0}^{\mathrm{BG}}(X),
$$

where $\omega(x)=\left[\phi_{x}\right]$.

Recall the map $\eta_{X}: \pi_{0}^{S}(X) \rightarrow \check{\pi}_{0}(X)$ given by $\eta_{X}([\alpha])=\check{\pi}_{0}(\alpha)(+\infty)$.

Definition 6.1. Let $X=(X, \phi, \varepsilon(X))$ be an exterior discrete semi-flow.

- An end $a \in \pi_{0}^{\mathrm{BG}}(X)$ is said to be $\omega$-representable if there is $x \in D(X)$ such that $\omega(x)=a$. Denote by $\omega_{0} \pi_{0}^{\mathrm{BG}}(X)$ the set of $\omega$-representable ends of $X$.

- An end $a \in \check{\pi}_{0}(X)$ is said to be $\check{\omega}$-representable if there is $x \in D(X)$ such that, for every $E \in \varepsilon(X)$, there is $n_{E} \in \mathbb{N}$ satisfying that, for every $n \geqslant n_{E}$, $\phi^{n}(x) \in C_{E}$, where $C_{E}$ is the path component of $a$ in $E$. Denote by ${ }^{\omega} \check{\pi}_{0}(X)$ the set of $\check{\omega}$-representable ends of $\check{\pi}_{0}(X)$ and by $\check{D}(X)$ the set of points $x \in D(X)$ such that there is $a \in \check{\pi}_{0}(X)$ satisfying that, for every $E \in \varepsilon(X)$, there is $n_{E}$ such that, for every $n \geqslant n_{E}, \phi^{n}(x) \in C_{E}$.

- An end $a=[\alpha] \in \pi_{0}^{S}(X)$, with $\alpha: \mathbb{R}_{+} \rightarrow X$ an exterior map, is said to be $\check{\omega}$ $\eta_{X}$-representable if $\eta_{X}(a)$ is $\check{\omega}$-representable. Denote by ${ }^{\omega} \pi_{0}^{S}(X)$ the set of $\check{\omega}-\eta_{X}$-representable ends of $\pi_{0}^{S}(X)$ and by $D^{S}(X)$ the set of points $x \in D(X)$ such that there is $a=[\alpha] \in \pi_{0}^{S}(X)$ such that $\left[\left.\alpha\right|_{\mathbb{N}}\right]=\left[\phi_{x}\right]$. 
From the definition of $\check{D}(X)$, it easy to see that there is an induced map

$$
\check{\omega}: \check{D}(X) \rightarrow \check{\pi}_{0}(X),
$$

which maps $x \in \check{D}(X)$ to the unique end point $a \in \check{\pi}_{0}(X)$ such that, for every $E \in$ $\varepsilon(X)$, there is $n_{E}$ satisfying that, for every $n \geqslant n_{E}, \phi^{n}(x) \in C_{E}$, where $C_{E}$ is the path component of $a$ in $E$.

Remark 6.2. Let $X=(X, \phi, \varepsilon(X))$ be an exterior discrete semi-flow which is firstcountable at infinity. Observe that we have a natural inclusion $L(X) \rightarrow D(X)$ (see Proposition 5.5). In general, the diagram

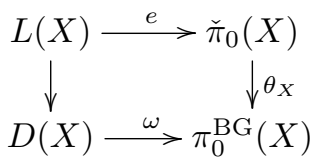

is not commutative. Nevertheless, the diagram commutes if we have an additional condition: "for every $x \in L(X)$ and for every $E \in \varepsilon(X)$, the points $x, \phi^{1}(x)$ are in the same path component of $E$ " (for instance, when $L(X) \subset \operatorname{Fix}(X)$ ). Note that, under this condition, $e$ agrees with $\check{\omega}$ on $\check{D}(X) \cap L(X)$ and the restriction to $\check{D}(X) \cap L(X)$ gives a commutative diagram.

Recall that the maps $\pi_{0}^{S}\left(\phi^{1}\right), \check{\pi}_{0}\left(\phi^{1}\right)$ and $\pi_{0}^{\mathrm{BG}}\left(\phi^{1}\right)$ induce on $\pi_{0}^{S}(X), \check{\pi}_{0}(X)$ and $\pi_{0}^{\mathrm{BG}}(X)$ the structure of a discrete semi-flow (set), respectively. In a similar way, ${ }^{\omega} \pi_{0}^{S}\left(\phi^{1}\right),{ }^{\omega} \check{\pi}_{0}\left(\phi^{1}\right)$ and ${ }^{\omega} \pi_{0}^{\mathrm{BG}}\left(\phi^{1}\right)$ induce on ${ }^{\omega} \pi_{0}^{S}(X),{ }^{\omega} \check{\pi}_{0}(X)$ and ${ }^{\omega} \pi_{0}^{\mathrm{BG}}(X)$ the structure of a discrete semi-flow (set), respectively.

In the following proposition, the analogues of the results of section 3 for representable ends are established and therefore they will be stated without proof:

Proposition 6.3. Let $X=(X, \phi, \varepsilon(X))$ be an exterior discrete semi-flow and consider the restrictions of the natural transformations $R_{X}$ and $\eta_{X}$ to the subsets of $\omega$-representable ends.

(i) In the following diagram

$$
\omega^{\omega} \pi_{0}^{\mathrm{S}}(X) \stackrel{{ }^{\omega} R_{X}}{\longrightarrow} \omega^{\omega} \pi_{0}^{\mathrm{BG}}(X) \stackrel{\mathrm{Id}}{\stackrel{{ }{ }_{S}}{\longrightarrow}} \omega \pi_{0}^{\mathrm{BG}}(X)
$$

the image of ${ }^{\omega} R$ is the equalizer of Id and ${ }^{\omega} S$.

(ii) If $X$ is first-countable at infinity, the following diagram is commutative

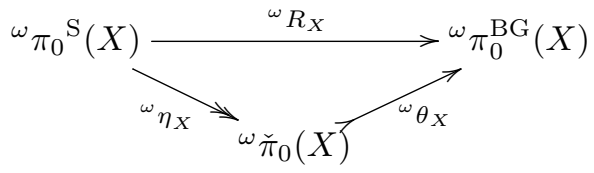

where ${ }^{\omega} R_{X}={ }^{\omega} \theta_{X} \circ{ }^{\omega} \eta_{X}$ is a canonical factorization of ${ }^{\omega} R_{X}$ as the composition of an epimorphism and a monomorphism. In this case, ${ }^{\omega} \check{\pi}_{0}(X)$ is the equalizer of Id and ${ }^{\omega} S$.

Now we study some relations between the different subsets of pseudo-attraction associated to an externology given in Definition 5.2 and the different types of representable end points. 
Proposition 6.4. Suppose that $X=(X, \phi, \varepsilon(X))$ is an exterior discrete semi-flow. Then:

(i) There are natural inclusions $D^{S}(X) \subset \check{D}(X) \subset D(X)$. Moreover, $\check{D}(X)$ is completely invariant and $D^{S}(X)$ is right-invariant.

(ii) The maps $\omega: D(X) \rightarrow \pi_{0}^{\mathrm{BG}}(X), \check{\omega}: \check{D}(X) \rightarrow \check{\pi}_{0}(X)$ are discrete semi-flow morphisms (between discrete semi-flow sets) which induce discrete semi-flow epimorphisms $\omega: D(X) \rightarrow{ }^{\omega} \pi_{0}^{\mathrm{BG}}(X), \check{\omega}: \check{D}(X) \rightarrow{ }^{\omega} \check{\pi}_{0}(X)$.

(iii) If $\pi_{0}\left(\phi^{1}\right)$ is injective, then ${ }^{\omega} \pi_{0}^{\mathrm{BG}}\left(\phi^{1}\right)$ is injective. If, in addition, $\phi^{1}$ is surjective, then ${ }^{\omega} \pi_{0}^{\mathrm{BG}}(X)$ has the structure of a discrete flow set.

(iv) The action induced on ${ }^{\omega} \check{\pi}_{0}(X)$ is trivial, that is, ${ }^{\omega} \check{\pi}_{0}(X) \subset \operatorname{Fix}\left(\check{\pi}_{0}(X)\right)$.

(v) $S \pi_{0}^{\mathrm{BG}}\left(\phi^{1}\right)=\pi_{0}^{\mathrm{BG}}\left(\phi^{1}\right) S,{ }^{\omega} \pi_{0}^{\mathrm{BG}}(X) \subset\left\{a \in \pi_{0}^{\mathrm{BG}}(X) \mid S(a)=\pi_{0}^{\mathrm{BG}}\left(\phi^{1}\right)(a)\right\}$.

Proof.

(i) It is straightforward to check.

(ii) Given $x \in D(X)$, we have $\phi_{\phi^{1}(x)}(n)=\phi^{1} \phi_{x}(n)$. Then $\omega\left(\phi^{1}(x)\right)=\left[\phi_{\phi^{1}(x)}\right]=$ $\left[\phi^{1} \phi_{x}\right]=\pi_{0}^{\mathrm{BG}}\left(\phi^{1}\right)\left[\phi_{x}\right]=\pi_{0}^{\mathrm{BG}}\left(\phi^{1}\right) \omega(x)$. There is a similar proof for $\check{\omega}$.

(iii) Suppose that $\pi_{0}^{\mathrm{BG}}\left(\phi^{1}\right)\left[\phi_{x}\right]=\pi_{0}^{\mathrm{BG}}\left(\phi^{1}\right)\left[\phi_{x^{\prime}}\right]$. Then we have that $\phi^{1}(x)$ is in the same path component as $\phi^{1}\left(x^{\prime}\right)$. Taking into account that $\pi_{0}\left(\phi^{1}\right)$ is injective, we have that there is a continuous path from $x$ to $x^{\prime}$. Using this path and an exterior homotopy from $\phi_{\phi^{1}(x)}^{1}$ to $\phi_{\phi^{1}\left(x^{\prime}\right)}^{1}$, we can construct a new exterior homotopy from $\phi_{x}$ to $\phi_{x^{\prime}}$.

Now suppose that $\phi^{1}$ is surjective. Then, given $y \in D(X)$, we can take $x \in X$ such that $\phi^{1}(x)=y$. Then $x \in D(X)$ and one has that $\pi_{0}^{\mathrm{BG}}\left(\phi^{1}\right)\left[\phi_{x}\right]=\left[\phi_{y}\right]$. This implies that $\pi_{0}^{\mathrm{BG}}\left(\phi^{1}\right)$ is surjective.

(iv) Just consider that $\check{\omega}\left(\phi^{1}(x)\right)=\check{\omega}(x), x \in \check{D}(X)$. It follows that the action is trivial.

(v) If $[\alpha] \in \pi_{0}^{\mathrm{BG}}(X)$, then $S \pi_{0}^{\mathrm{BG}}\left(\phi^{1}\right)[\alpha]=\left[\left(\phi^{1} \alpha\right) s\right]=\left[\phi^{1}(\alpha s)\right]=\pi_{0}^{\mathrm{BG}}\left(\phi^{1}\right) S[\alpha]$. Besides, if $x \in D(X)$, then $S \omega(x)=S\left[\phi_{x}\right]=\left[\phi^{1} \phi_{x}\right]=\pi_{0}^{\mathrm{BG}}\left(\phi^{1}\right) \omega(x)$.

Now we introduce the basins of end points and we consider special end points whose basins are open subsets of $X$. Some local stability notions with respect to end points are also considered.

Definition 6.5. Given an exterior discrete semi-flow $X=(X, \phi, \varepsilon(X))$ and an element $a \in{ }^{\omega} \pi_{0}^{\mathrm{BG}}(X)$, the subspace $\omega^{-1}(a)$, denoted by $D_{a}$, will be called the $\omega$-basin of $a$. Similarly, given an element $a \in{ }^{\omega} \check{\pi}_{0}(X)$, the subspace $\check{\omega}^{-1}(a)$, denoted by $\check{D}_{a}$, will be called the $\check{\omega}$-basin of $a$.

When $a \in{ }^{\omega} \pi_{0}^{S}(X)$, the subspace $D_{a}^{S}=D^{S}(X) \cap \check{D}_{\eta_{X}(a)}$ will be called the immediate basin of $a$.

Definition 6.6. Let $X$ be an exterior discrete semi-flow.

(i) An end point $a \in{ }^{\omega} \pi_{0}^{\mathrm{BG}}(X)$ is said to be an $\omega$-attractor if its basin $D_{a}$ is an open subset of $X$. We write ${ }_{A}^{\omega} \pi_{0}^{\mathrm{BG}}(X)=\left\{a \in{ }^{\omega} \pi_{0}^{\mathrm{BG}}(X) \mid a\right.$ is an $\omega$-attractor $\}$ and ${ }_{N}^{\omega} \pi_{0}^{\mathrm{BG}}(X)={ }^{\omega} \pi_{0}^{\mathrm{BG}}(X) \backslash{ }_{A}^{\omega} \pi_{0}^{\mathrm{BG}}(X)$.

(ii) An end point $a \in{ }^{\omega} \check{\pi}_{0}(X)$ is said to be an $\check{\omega}$-attractor if its basin $\check{D}_{a}$ is an open subset of $X$. We write ${ }_{A}^{\omega} \check{\pi}_{0}(X)=\left\{a \in{ }^{\omega} \check{\pi}_{0}(X) \mid a\right.$ is an $\check{\omega}$-attractor $\}$ and ${ }_{N}^{\omega} \check{\pi}_{0}(X)={ }^{\omega} \check{\pi}_{0}(X) \backslash \stackrel{\omega}{A}_{0} \check{\pi}_{0}(X)$. 
(iii) A point $x \in D(X)$ is locally $\omega$-stable if there is an open neighborhood $U$ such that $U \subset D(X)$ and, for every $y \in U, \omega(y)=\omega(x)$.

(iv) A point $x \in \check{D}(X)$ is locally $\check{\omega}$-stable if there is an open neighborhood $U$ such that $U \subset \check{D}(X)$ and, for every $y \in U, \check{\omega}(y)=\check{\omega}(x)$.

We have the following basic properties which are immediately deduced from the definitions:

Lemma 6.7. Let $X=(X, \phi, \varepsilon(X))$ be an exterior discrete semi-flow and let $x \in$ $D(X)$. The following statements hold:

(i) If the end $\omega(x)$ is an $\omega$-attractor, then $x$ is locally $\omega$-stable.

(ii) If $a \in{ }^{\omega} \pi_{0}^{\mathrm{BG}}(X)$ and $x \in D_{a}, x$ is locally $\omega$-stable if and only if $x \in \stackrel{\circ}{D}_{a}$. If, for every $y \in D_{a}, y$ is locally $\omega$-stable, then a is an $\omega$-attractor.

(iii) If the end $\check{\omega}(x)$ is an $\check{\omega}$-attractor, then $x$ is locally $\check{\omega}$-stable.

(iv) If $a \in \omega^{\check{\pi}_{0}}(X)$ and $x \in \check{D}_{a}, x$ is locally $\check{\omega}$-stable if and only if $x \in \check{D}_{a}$. If, for every $y \in \check{D}_{a}$, y is locally $\check{\omega}$-stable, then a is an $\check{\omega}$-attractor.

The maps $\omega$ and $\check{\omega}$ permit us to divide an exterior discrete semi-flow:

Corollary 6.8. Let $X=(X, \phi, \varepsilon(X))$ be an exterior discrete semi-flow and denote $D=D(X), \check{D}=\check{D}(X), D^{S}=D^{S}(X)$. Then we have the following induced partitions of $X$ :

$$
\begin{aligned}
& X=(X \backslash D) \sqcup\left(\bigsqcup_{a \in \in^{\omega} \pi_{0}^{\mathrm{BG}}(X)} D_{a}\right), \\
& X=(X \backslash \check{D}) \sqcup\left(\bigsqcup_{a \in \omega \check{\pi}_{0}(X)} \check{D}_{a}\right) \text {, } \\
& X=\left(X \backslash D^{S}\right) \sqcup\left(\bigsqcup_{a \in \omega^{\omega} \pi_{0}^{S}(X)} D_{a}^{S}\right),
\end{aligned}
$$

where $X \backslash D, X \backslash \check{D}$ and $\check{D}_{a}$ are completely invariant and $D_{a}^{S}$ is right-invariant.

In general $D_{a}$ is not right invariant. However, some notable unions of these basins are completely invariant subsets:

Proposition 6.9. Let $X=(X, \phi, \varepsilon(X))$ be an exterior discrete semi-flow. Then:

(i) If $a \in{ }^{\omega} \pi_{0}^{\mathrm{BG}}(X)$ and $[a]$ is the completely invariant big orbit (see subsection 2.2) of a in ${ }^{\omega} \pi_{0}^{\mathrm{BG}}(X)$, then $\bigsqcup_{a^{\prime} \in[a]} D_{a^{\prime}}$ is completely invariant.

(ii) If $\pi_{0}\left(\phi^{1}\right)$ is injective and $\phi^{1}: X \rightarrow X$ is a surjective open map, then ${ }_{A}^{\omega} \pi_{0}^{\mathrm{BG}}(X)$ is completely invariant in ${ }^{\omega} \pi_{0}^{\mathrm{BG}}(X), \bigsqcup_{a \in \in_{A}^{\omega} \pi_{0}^{\mathrm{BG}}(X)} D_{a}$ is an open completely invariant subset of $X$ and $\bigsqcup_{a \in \in_{N}^{\omega} \pi_{0}^{\mathrm{BG}}(X)} D_{a}$ is completely invariant and closed in $D(X)$.

(iii) If $X$ is first-countable at infinity, then the following diagram is commutative:

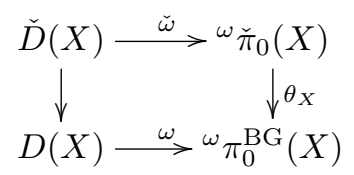


Proof. A simple inspection proves (i) and (iii).

It only remains to check (ii). By Proposition 6.4(ii)-(iii), we have a commutative diagram

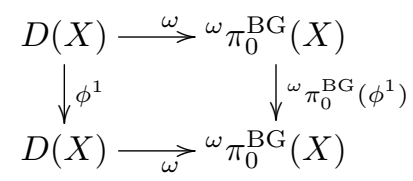

where ${ }^{\omega} \pi_{0}^{\mathrm{BG}}\left(\phi^{1}\right)$ is bijective. So $\omega^{-1}\left({ }^{\omega} \pi_{0}^{\mathrm{BG}}\left(\phi^{1}\right)(a)\right)=\phi^{1}\left(\omega^{-1}(a)\right)$, for $a \in$ $\omega^{\omega} \pi_{0}^{\mathrm{BG}}(X)$. Since $\phi^{1}$ is a continuous open map, it follows that ${ }_{A}^{\omega} \pi_{0}^{\mathrm{BG}}(X)$ is completely invariant and also gives the rest of the assertion.

Using the subset of end points which are attractors and its complement, under the conditions given in Proposition 6.9, one can divide the region of pseudo-attraction of the externology as the union of a completely invariant open subset and its complement:

Definition 6.10. Given an exterior discrete semi-flow $X=(X, \phi, \varepsilon(X))$,

$$
{ }_{A} D(X)=\bigsqcup_{a \in \in_{A}^{\omega} \pi_{0}^{\mathrm{BG}}(X)} D_{a}, \quad{ }_{N} D(X)=\bigsqcup_{a \in \in_{N}^{\omega} \pi_{0}^{\mathrm{BG}}(X)} D_{a}
$$

are the ${ }^{\omega} \pi_{0}^{\mathrm{BG}}$-attracting basin and the ${ }^{\omega} \pi_{0}^{\mathrm{BG}}$-non-attracting basin of $X$, respectively. Similarly,

$$
{ }_{A} \check{D}(X)=\bigsqcup_{a \in \epsilon_{A}^{\omega} \check{\pi}_{0}(X)} \check{D}_{a}, \quad{ }_{N} \check{D}(X)=\bigsqcup_{a \in \in_{N}^{\omega} \check{\pi}_{0}(X)} \check{D}_{a}
$$

are the ${ }^{\omega} \check{\pi}_{0}$-attracting basin and the ${ }^{\omega} \check{\pi}_{0}$-non-attracting basin of $X$.

The intersection with the limit sets

$$
\begin{aligned}
& { }_{A} L(X)={ }_{A} D(X) \cap L(X), \quad{ }_{N} L(X)={ }_{N} D(X) \cap L(X), \\
& { }_{A} \check{L}(X)={ }_{A} \check{D}(X) \cap L(X), \quad{ }_{N} \check{L}(X)={ }_{N} \check{D}(X) \cap L(X)
\end{aligned}
$$

are the corresponding attracting (non-attracting) limit of $X$.

Remark 6.11. In a similar way, we can consider canonical maps

$$
\bar{\omega}: \bar{D}(X) \rightarrow \bar{\pi}_{0}^{\mathrm{BG}}(X),
$$

and basins of the form

$$
\bar{D}_{a}=\bar{\omega}^{-1}(a), \quad a \in{ }^{\omega} \bar{\pi}_{0}^{\mathrm{BG}}(X) .
$$

The new sets of end points and bar-limits can be divided with the corresponding notions of attracting end points. The authors suggest that subsets of the following type

$$
\begin{aligned}
& { }_{N} \bar{L}(X)={ }_{N} \bar{D}(X) \cap \bar{L}(X), \\
& { }_{N} \check{L}(X)={ }_{N} \overline{\bar{D}}(X) \cap \bar{L}(X)
\end{aligned}
$$

can be used to give pure topological analogues of Julia sets in a topological semi-flow. 


\section{The externology of right-absorbing open subsets}

For a given discrete semi-flow, $X=(X, \phi)$, we can consider the externology $\varepsilon^{\mathbf{r}}(X)$ given by all the open subsets $E$ such that, for every $x \in X$, there is $n \in \mathbb{N}$ satisfying that, for $m \geqslant n, \phi^{m}(x) \in E$. For this externology, one has that $\left(X, \phi, \varepsilon^{\mathbf{r}}(X)\right)$ is a d-exterior discrete semi-flow and therefore we have that $D(X)=X$.

Definition 7.1. For a given discrete semi-flow $(X, \phi)$, the externology $\varepsilon^{\mathbf{r}}(X)$ is said to be the right-absorbing externology of $(X, \phi)$ (or just the right externology) and an open $E \in \varepsilon^{\mathbf{r}}(X)$ is said to be an $\mathbf{r}$-exterior open subset of $X$.

In this section we consider the following basic properties:

Lemma 7.2. Let $(X, \phi, \varepsilon(X))$ be an exterior discrete semi-flow. Then the following statements are equivalent:

(i) $\varepsilon(X) \subset \varepsilon^{\mathbf{r}}(X)$.

(ii) $(X, \phi, \varepsilon(X))$ is a d-exterior discrete semi-flow.

(iii) $D(X)=X$.

It is important to note that one has a canonical functor $\mathbf{F}(\mathbb{N}) \rightarrow \mathbf{E}^{\mathrm{d}} \mathbf{F}(\mathbb{N})$ which carries $(X, \phi)$ to $\left(X, \phi, \varepsilon^{\mathbf{r}}(X)\right)$. We will use the reduced notation $X=(X, \phi)$ and $X^{\mathbf{r}}=\left(X, \phi, \varepsilon^{\mathbf{r}}(X)\right)$. Using this canonical construction all the different constructions (limits, end sets, etc.) given for exterior discrete semi-flows can be applied to a discrete semi-flow.

In the next subsections, some relations between constructions associated to an exterior space and dynamic properties of a discrete semi-flow are analyzed. In particular, we see the relation between limits and periodic points, as well as between bar-limits and omega-limits.

\subsection{Periodic points}

The relation of the limit space of an exterior discrete semi-flow and the sub-flow of periodic points is analyzed in the following results.

Lemma 7.3. If $X=(X, \phi, \varepsilon(X))$ is a $\mathbf{d}$-exterior discrete semi-flow, then $P(X) \subset$ $L(X)$. In particular, if $X$ is a discrete semi-flow, then $P(X) \subset L\left(X^{\mathbf{r}}\right)$.

Proof. Take a periodic point $x$ and an arbitrary $E \in \varepsilon(X)$. Since $\phi_{x}: \mathbb{N} \rightarrow X$ is exterior we have that $\phi_{x}^{-1}(E)$ is an exterior subset of $\mathbb{N}$. In other words, there exists $n \in \mathbb{N}$ such that $(n,+\infty) \cdot x \subset E$. From the fact that $x$ is periodic, we have the equality $(n,+\infty) \cdot x=\mathbb{N} \cdot x$ and, taking into account that $x \in \mathbb{N} \cdot x$, we conclude that $x \in E$.

Lemma 7.4. Let $X$ be a discrete semi-flow and suppose that $X$ is a $T_{1}$-space. Then, for every $x \in X$, the following statements are equivalent:

(i) $x$ is a non-periodic point.

(ii) $X \backslash\{x\}$ is an $\mathbf{r}$-exterior open subset of $X$.

Proof. In order to prove that (i) implies (ii), take $y \in X$; if $(\mathbb{N} \cdot y) \cap(\mathbb{N} \cdot x)=\emptyset$, then, for every $n \in \mathbb{N},(n,+\infty) \cdot y \subset X \backslash\{x\}$. If $(\mathbb{N} \cdot y) \cap(\mathbb{N} \cdot x) \neq \emptyset$, considering that $x$ is 
not periodic, one can find $n \in \mathbb{N}$ such that $(n,+\infty) \cdot y \subset X \backslash\{x\}$. Then one has that $X \backslash\{x\} \in \varepsilon^{\mathbf{r}}(X)$. Conversely, suppose that $x$ is a periodic point. Then, by Lemma 7.3 above, $X \backslash\{x\}$ is not $\mathbf{r}$-exterior.

Using these two lemmas, we obtain the following result:

Theorem 7.5. Let $X$ be a discrete semi-flow and suppose that $X$ is a $T_{1}$-space. Then

$$
L\left(X^{\mathbf{r}}\right)=P(X) .
$$

Proof. Let $x \in X \backslash P(X)$. Then, by Lemma 7.4, one has that $X \backslash\{x\} \in \varepsilon^{\mathbf{r}}(X)$ and

$$
P(X)=X \backslash\left(\bigcup_{x \notin P(X)}\{x\}\right)=\bigcap_{x \notin P(X)} X \backslash\{x\} \supset \bigcap_{E \in \varepsilon^{\mathbf{r}}(X)} E=L\left(X^{\mathbf{r}}\right) .
$$

Now, the result follows from Lemma 7.3.

Taking into account the theorem above, if $X$ is a $T_{1}$ discrete semi-flow, in the diagram of distinguished sub-flows

$$
P(X) \subset \operatorname{Poisson}(X) \subset \Lambda\left(X^{\mathbf{r}}\right)
$$

we have identified the first sub-flow as a limit set:

$$
L\left(X^{\mathbf{r}}\right)=P(X) \subset \operatorname{Poisson}(X) \subset \Lambda\left(X^{\mathbf{r}}\right) \subset X .
$$

\subsection{Limits and omega-limits}

In the following result we analyze the relationship between the omega-limit and the bar-limit induced by an externology.

Lemma 7.6. If $X=(X, \phi, \varepsilon(X))$ is an exterior discrete semi-flow, then

$$
\Lambda(D(X)) \subset \Lambda(\bar{D}(X)) \subset \overline{\Lambda(\bar{D}(X))} \subset \bar{L}(X) .
$$

Proof. If $E \in \varepsilon(X)$, then, for every $x \in \bar{D}(X)$, there exists $n \in \mathbb{N}$ such that $(n,+\infty)$. $x \subset \bar{E}$ and therefore $\overline{(n,+\infty) \cdot x} \subset \bar{E}$. By definition, this implies that $\Lambda(x) \subset \bar{L}(X)$, for every $x \in \bar{D}(X)$. Hence, $\Lambda(\bar{D}(X)) \subset \bar{L}(X)$. Taking into account that $\bar{L}(X)$ is a closed subset, we also have $\overline{\Lambda(\bar{D}(X))} \subset \bar{L}(X)$.

And now let us present some technical results:

Lemma 7.7. Let $(X, \varepsilon(X))$ be an exterior space and $x \in X$. Then there exists an open neighborhood $V_{x}$ at $x$ such that $X \backslash \bar{V}_{x} \in \varepsilon(X)$ if and only if $x \notin \bar{L}(X)$.

Proof. If $X \backslash \bar{V}_{x} \in \varepsilon(X)$, then, taking into account that $V_{x} \cap\left(X \backslash \bar{V}_{x}\right)=\emptyset$, we have that $x \notin \overline{X \backslash \bar{V}_{x}}$. Therefore we have that $x \notin \bigcap_{E \in \varepsilon(X)} \bar{E}=\bar{L}(X)$.

Conversely, if $x \notin \bar{L}(X)$, then there exists $E \in \varepsilon(X)$ such that $x \notin \bar{E}$. Now, taking $V_{x}=X \backslash \bar{E}=\operatorname{Int}(X \backslash E)$, we have that $X \backslash \bar{V}_{x}=\operatorname{Int}\left(X \backslash V_{x}\right)=\operatorname{Int}(\bar{E}) \supset E$. Consequently, $X \backslash \bar{V}_{x} \in \varepsilon(X)$.

Lemma 7.8. Let $X=(X, \phi, \varepsilon(X))$ be an exterior discrete semi-flow and let $x$ be in $X$. If there exists an open neighborhood $V_{x}$ at $x$ such that $X \backslash \bar{V}_{x} \in \varepsilon(X)$, then $x \notin$ $\overline{\Lambda(\bar{D}(X))}$. 
Proof. This is a consequence of Lemma 7.7 and Lemma 7.6.

Proposition 7.9. Let $X$ be a discrete semi-flow and $x \in X$. If there exists an open neighborhood $V_{x}$ at $x$ such that $X \backslash \bar{V}_{x}$ is $\mathbf{r}$-exterior, then $x \notin \overline{\Lambda(X)}$.

Proof. This is a consequence of Lemma 7.8 and Lemma 7.2.

Lemma 7.10. Let $X$ be a discrete semi-flow which is a locally compact regular space. If $x \notin \overline{\Lambda(X)}$, then there exists an open neighborhood $V_{x}$ at $x$ such that $X \backslash \bar{V}_{x}$ is $\mathbf{r}$ exterior.

Proof. Suppose that $x \notin \overline{\Lambda(X)}$. Since $X$ is locally compact, there is a compact neighborhood $K$ at $x$ such that $K \cap \Lambda(X)=\emptyset$. Take $y \in X$ and assume that, for every $m \in \mathbb{N},(m,+\infty) \cdot y \cap K \neq \emptyset$. Then there is a sequence $m_{n} \rightarrow+\infty$ such that $m_{n} \cdot y \in$ $K$. As $K$ is compact, one can take a sub-net $m_{n_{i}} \rightarrow+\infty$ such that $m_{n_{i}} \cdot y \rightarrow u \in K$. This fact implies that $u \in K \cap \Lambda(y) \subset K \cap \Lambda(X)$, which is a contradiction. Therefore, there is $m \in \mathbb{N}$ such that $(m,+\infty) \cdot y \cap K=\emptyset$. By the regularity of $X$, there exists an open neighborhood $V_{x}$ at $x$ such that $\bar{V}_{x} \subset K$ and $X \backslash \bar{V}_{x}$ is r-exterior.

Corollary 7.11. Let $X$ be a discrete semi-flow. If $X$ is a locally compact regular space, then $\bar{L}\left(X^{\mathbf{r}}\right) \subset \overline{\Lambda(X)}$.

Proof. If $x \notin \overline{\Lambda(X)}$, by the lemma above there exists an open neighborhood $V_{x}$ at $x$ such that $X \backslash \bar{V}_{x}$ is r-exterior. By Lemma 7.7, it follows that $x \notin \bar{L}\left(X^{\mathbf{r}}\right)$.

By the corollary above and Lemma 7.6, we obtain the following result:

Theorem 7.12. Let $X$ be a discrete semi-flow. If $X$ is a locally compact regular space, then $\bar{L}\left(X^{\mathbf{r}}\right)=\overline{\Lambda(X)}$.

Corollary 7.13. Let $X$ be a discrete semi-flow. If $X$ is a locally compact $T_{3}$ space, then

$$
L\left(X^{\mathbf{r}}\right)=P(X) \subset \operatorname{Poisson}(X) \subset \Lambda(X) \subset \overline{\Lambda(X)}=\bar{L}\left(X^{\mathbf{r}}\right) .
$$

Proof. This is a consequence of Theorem 7.5 and Theorem 7.12.

\section{Other externologies and examples}

If we take a right-invariant subset $S$ of a discrete semi-flow $X$, new families of externologies can be analyzed: for instance, we can consider the externology $\epsilon(X, S)$ formed by all the open neighborhoods of $S$ in $X$.

As an example of the notions and constructions developed in this paper, we analyze the decompositions given by the externologies $\epsilon\left(X, P_{1}\right)$ and $\epsilon\left(X, P_{2}\right)$ induced by the right-invariant subsets of 1-periodic (fixed) points $P_{1}$ and 2-periodic points $P_{2}$, respectively, when we consider on the Riemann sphere $X=\mathbb{C} \cup\{\infty\}$ the discrete semi-flow induced by the polynomial function $h(z)=z^{2}-1(h(\infty)=\infty)$.

The dynamics of quadratic polynomials has been profusely studied through techniques and notions of complex dynamics. In the following paragraphs, for the particular case $(X, h)$, we express the regions of attraction of the externologies as union of attraction basins and the set of Brown-Grossman ends as subsets of periodic points.

The fixed points of $h$ are $\infty$ and the two golden numbers $p_{1}=\frac{1-\sqrt{5}}{2}$ and $p_{2}=\frac{1+\sqrt{5}}{2}$. 
If we consider the exterior discrete semi-flow $X_{1}=\left(\mathbb{C} \cup\{\infty\}, h, \epsilon\left(X, P_{1}\right)\right)$, we have the induced map

$$
\omega_{1}: D\left(X_{1}\right) \rightarrow{ }^{\omega} \pi_{0}^{\mathrm{BG}}\left(X_{1}\right)
$$

from the region of attraction $D\left(X_{1}\right)$ to the set of omega-representable end points of Brown-Grossman type. In this case, one has a canonical isomorphism ${ }^{\omega} \pi_{0}^{\mathrm{BG}}\left(X_{1}\right) \cong P_{1}$.

The externology $\epsilon\left(X, P_{1}\right)$ induces a decomposition of the following form:

$$
X=\left(X \backslash D\left(X_{1}\right)\right) \sqcup \omega_{1}^{-1}(\infty) \sqcup \omega_{1}^{-1}\left(p_{1}\right) \sqcup \omega_{1}^{-1}\left(p_{2}\right),
$$

which can be seen in the Figure 1 .
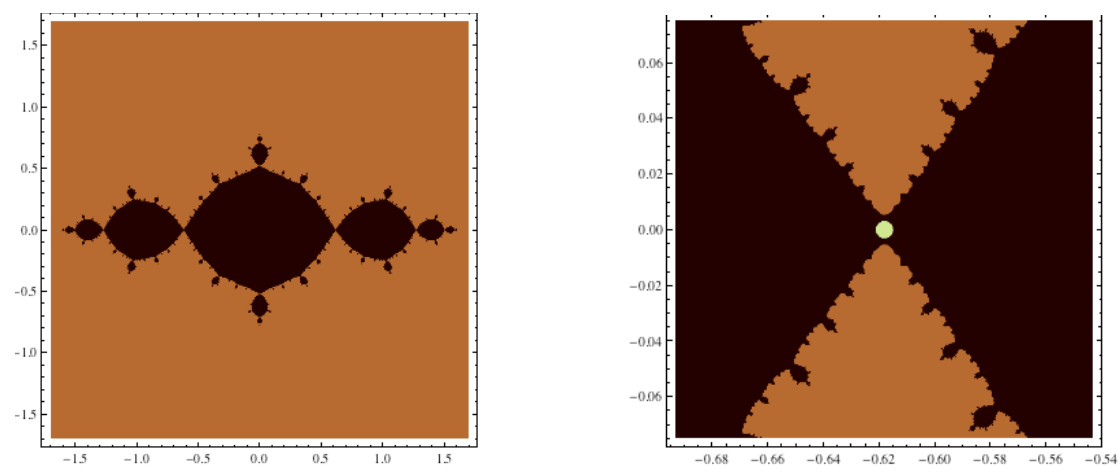

Figure 1: On the left, the basin of the attracting infinity point in brown (grey in the printed version) and, on the right, a point of the basin of the repelling fixed point $p_{1}$.

The basin of $\infty$ is displayed using brown color (grey in the printed version), the golden numbers are repulsing points and their basins are not visible in the figure on the left. Nevertheless, when we apply a zoom effect, it is possible to see some points in the basins of the end points associated to the golden numbers: the central point in the figure on the right is a point of the basin of a golden number $p_{1}$. The black color corresponds to points which are not in basins of fixed points; that is, to $X \backslash D\left(X_{1}\right)$.

When the 2-periodic points of $h$ are studied, one obtains: $P_{2}=\left\{\infty,-1, p_{1}, 0, p_{2}\right\}$. For the exterior discrete semi-flow $X_{2}=\left(\mathbb{C} \cup\{\infty\}, h, \epsilon\left(X, P_{2}\right)\right)$ and the induced map

$$
\omega_{2}: D\left(X_{2}\right) \rightarrow{ }^{\omega} \pi_{0}^{\mathrm{BG}}\left(X_{2}\right)
$$

from the region of attraction $D\left(X_{2}\right)$ to the set of omega-representable end points of Brown-Grossman type, one has a canonical isomorphism ${ }^{\omega} \pi_{0}^{\mathrm{BG}}\left(X_{2}\right) \cong P_{2}$. For $X_{2}$, it is interesting to check that ${ }^{\omega} \check{\pi}_{0}\left(X_{2}\right) \cong{ }^{\omega} \pi_{0}^{S}\left(X_{2}\right) \cong P_{1}$. The cyclic point -1 generates the Brown-Grossman end point represented by the sequence $(-1,0,-1,0, \ldots)$ and the cyclic point 0 induces the end point represented by $(0,-1,0,-1 \ldots)$.

To compare the decompositions induced by $P_{1}$ and $P_{2}$, one has to analyze the diagram

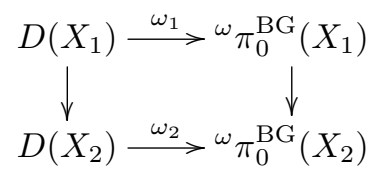


where $D\left(X_{1}\right) \rightarrow D\left(X_{2}\right)$ is the inclusion:

$$
\omega_{1}^{-1}(\infty) \sqcup \omega_{1}^{-1}\left(p_{1}\right) \sqcup \omega_{1}^{-1}\left(p_{2}\right) \subset \omega_{2}^{-1}(\infty) \sqcup \omega_{2}^{-1}(-1) \sqcup \omega_{2}^{-1}\left(p_{1}\right) \sqcup \omega_{2}^{-1}(0) \sqcup \omega_{2}^{-1}\left(p_{2}\right) .
$$

It is interesting to note that, in Figure 2, the black part associated to $\omega_{1}$ has been divided into the union of the basins of the new 2-cyclic attracting points of $h$. The immediate basins $D_{-1}^{S}$ and $D_{0}^{S}$ form the path component of the basin containing the 2-cyclic points -1 and 0 , respectively.

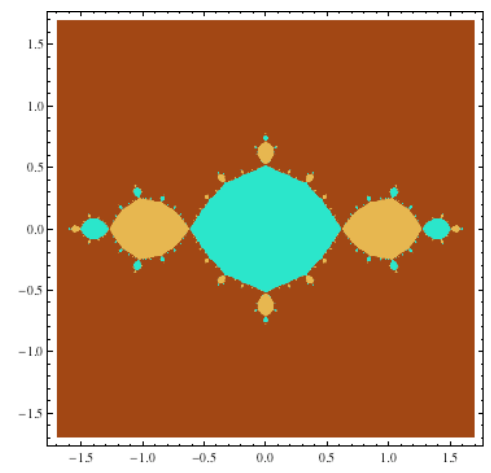

Figure 2: The basins of the new 2-cyclic points correspond to the black part of Figure 1.
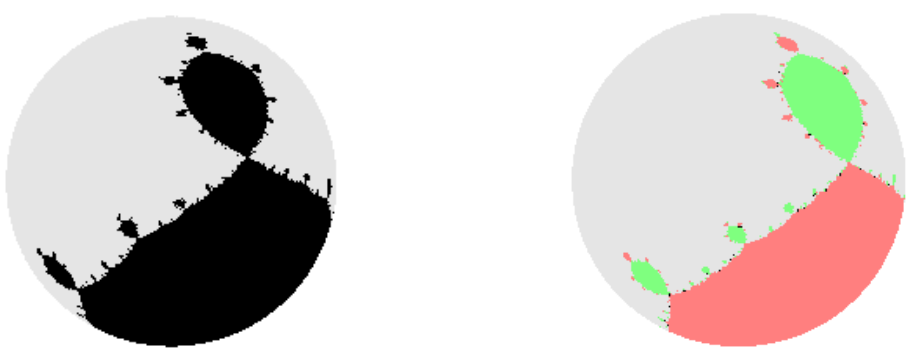

Figure 3: The basins of $D\left(X_{1}\right)$ and $D\left(X_{2}\right)$ with new colors (mid and dark grey in the printed version) on the Riemann sphere.

Remark 8.1. The figures of this section have been obtained by using graphic tools of a computational project developed by the authors (see [7]) which studies attraction basins of branched self-coverings.

\section{Future applications}

The authors think that it would be interesting to develop a deeper study of externologies associated to a right-invariant subspace $S$ of a discrete semi-flow $X$ given by all the open neighborhoods of $S$ in $X$.

The results obtained for the example given in the above section suggest that some 
of the particular properties analyzed can be proven for more general discrete semiflows. For externologies given by open neighborhoods of an invariant subset $S$, one can prove that, under good conditions, the region of attraction of the externology is the region of attraction of $S$. In some cases, the limit of the externology agrees with $S$ and the set of end points of Borsuk-Cech type is related to the connected components of $S$. Moreover, the omega-representable end points of Borsuk-Čech type are related to the fixed points of $S$, and the basins of end points, under suitable conditions, correspond to basins of fixed points. The basin of a representable end point of Steenrod type is related to the immediate component of the basin of a fixed point. The basins of omega-representable end points of Brown-Grossman type are related to the basins of periodic points which are contained in $S$.

It is worth pointing out that the open neighborhoods of a subset $S$ on a manifold can also be taken as a resolution (in the sense of shape theory) of $S$ and this fact gives the possibility of applying techniques of shape theory, like shape Conley index (see $[1,2,28]$ ), algebraic characterization of shape equivalences, etc. We can also use these externologies/resolutions to compare techniques and results coming from exterior homotopy theory and from shape theory. For instance, exterior spaces can also be used for the study of the exterior homotopy type of attractors and repellers of discrete and continuous flows and to compare them with their corresponding shapes, see $[22,23,29,30]$.

The study of semi-flows of the form $(M, h)$, where $M$ is a manifold and $h: M \rightarrow M$ is a branched covering (see [7]), can be also analyzed with the techniques developed in this paper, by either taking externologies given by open neighborhoods of suitable subsets of periodic points or taking the right-absorbing externology. Many questions arise, such as the study of Brown-Grossman end points associated to points in the singular subset of the branched covering.

The discretization processes (the first return Poincaré map, the discretization of a continuous semi-flow, etc.) and anti-discretization constructions (suspension, prolongation and telescopes, see [9]) can also be analyzed by means of externologies associated to continuous and discrete semi-flows. This will permit us to connect the theory of basins of end points developed in [16] for continuous flows with the basins of end points of discrete semi-flows.

Finally, it is important to say that the problem of sensitivity with respect to the initial conditions can be studied by taking the externology of open subsets that capture the "future part" of "tubes" generated by trajectories of neighborhoods at each point.

\section{References}

[1] H. Barge and J.M.R. Sanjurjo, Regular blocks and Conley index of isolated invariant continua in surfaces Nonlinear Anal., 146, 100-119 (2016).

[2] H. Barge and J.M.R. Sanjurjo, Fixed points, bounded orbits and attractors of planar flows, A mathematical tribute to Professor José María Montesinos Amilibia, 125-132 (2016).

[3] G.D. Birkhoff, Dynamical Systems, Amer. Math. Soc. Colloq. Publ., vol. 9, 1927. 
[4] A. Del Río, L.J. Hernández and M.T. Rivas Rodríguez, S-Types of global towers of spaces an exterior spaces, Appl. Categ. Structures 17, no. 3, 287-301 (2009).

[5] D. Edwards and H. Hastings, Čech and Steenrod homotopy theories with applications to geometric topology, Lecture Notes in Math., vol. 542, Springer-Verlag, Berlin, Heidelberg, New York, 1976.

[6] J.I. Extremiana, L.J. Hernández and M.T. Rivas, An isomorphism theorem of the Hurewicz Type in the proper homotopy category, Fund. Math., 132, 195-214 (1989).

[7] J.I. Extremiana, L.J. Hernández and M.T. Rivas, Self-overlays and symmetries of Julia sets of expanding maps, Rev. R. Acad. Cienc. Exactas Fís. Nat. Ser. A Mat. RACSAM 112, no. 3, 829-848 (2018).

[8] J.I. Extremiana, L.J. Hernández and M.T. Rivas, Postnikov factorizations at infinity, Topology Appl., 153, 370-393 (2005).

[9] J.M. Fernández-Cestau, L.J. Hernández-Paricio and M.T. Rivas-Rodríguez, Prolongations, Suspensions and Telescopes, Appl. Categ. Structures, 25, no. 5, 709-745 (2017). https://doi.org/10.1007/s10485-016-9427-1.

[10] H. Freudenthal, Über die Enden topologisher Räume und Gruppen, Math. Z., 53, 692-713 (1931).

[11] J.M. García Calcines, Homotopía propia simplicial, Tesis, La Laguna, 1998.

[12] J.M. García-Calcines, P.R. García-Díaz and A. Murillo, A Whitehead-Ganea approach for proper Lusternik-Schnirelmann category, Math. Proc. Cambridge Philos. Soc., 142, no. 3, 439-457 (2007).

[13] J.M. García-Calcines, P.R. García-Díaz and A. Murillo, The Ganea conjecture in proper homotopy via exterior homotopy theory, Math. Proc. Cambridge Philos. Soc., 149, 75-91 (2010).

[14] J.M. García Calcines, M. García Pinillos and L.J. Hernández, A closed simplicial model category for proper homotopy and shape theories, Bull. Aus. Math. Soc., 57, no. 2, 221-242 (1998).

[15] J.M. García Calcines, M. García Pinillos and L.J. Hernández, Closed simplicial model structures for exterior and proper homotopy, Appl. Categ. Structures, 12, no. 3, 225-243 (2004).

[16] J.M. García Calcines, L.J. Hernández and M.T. Rivas Rodríguez, Limit and end functors of dynamical systems via exterior spaces, Bull. Belg. Math. Soc. Simon Stevin, 20, 937-959 (2013).

[17] J.M. García Calcines, L.J. Hernández and M.T. Rivas Rodríguez, A completion construction for continuous dynamical systems, Topol. Methods Nonlinear Anal., 44, no. 2, 497-526 (2014).

[18] J.M. García Calcines, L.J. Hernández and M.T. Rivas Rodríguez, Omega Limits, Prolongational Limits and Almost Periodic Points of a Continuous Flow via Exterior Spaces, Glas. Mat. Ser. III, 52, no. 2, 295-329 (2017).

[19] M. García Pinillos, L.J. Hernández Paricio and M.T. Rivas Rodríguez, Exact sequences and closed model categories, Appl. Categ. Structures, 18, no. 4, 343375 (2010). 
[20] L.J. Hernández Paricio and T. Porter, Global analogues of the Brown-Grossman proper homotopy groups of an end, Math. Proc. Cambridge Philos. Soc., 104, 483-496 (1988).

[21] S. Mardešić and J. Segal. Shape Theory. North-Holland, Amsterdam, 1982.

[22] M.A. Morón and F.R. Ruiz del Portal, A note about the shape of attractors of discrete semi-dynamical systems, Proc. Amer. Math. Soc., 134, 2165-2167 (2006).

[23] M.A. Morón, J.J. Sánchez Gabites and J.M.R. Sanjurjo, Topology and dynamics of unstable attractors, Fund. Math., 197, 239-252 (2007).

[24] H. Poincaré, Les méthodes nouvelles de la mécanique céleste, Paris, GauthierVillars et fils, 1892-99, 1892.

[25] H. Poincaré, Mémoire sur les courbes définies par une équation différentielle, Handbook of Algebraic Topology, Chapter 3, pp. 127-167, 1995.

[26] T. Porter, Čech and Steenrod homotopy and the Quigley exact couple in strong shape and proper homotopy theory, J. Pure Appl. Algebra, 24, 303312 (1983).

[27] T. Porter, Proper homotopy theory, Handbook of algebraic topology, NorthHolland, Amsterdam, pp. 127-167, 1995.

[28] J.W. Robbin and D. Salamon, Dynamical systems, shape theory and the Conley index, Ergodic Theory Dynam. Systems, 8 (Charles Conley Memorial Volume), 375-393 (1988).

[29] J.J. Sánchez Gabites, Dynamical systems and shapes, Rev. R. Acad. Cienc. Exactas Fís. Nat. Ser. A Math. RACSAM, 102, no. 1, 127-160 (2008).

[30] J.M. Sanjurjo, Stability, attraction and shape; a topological study of flows, Lect. Notes Nonlinear Anal., vol. 12, 93-122 (2011).

J.M. García Calcines jmgarcal@ull.es

Dpto. de Matemáticas, Estad. e I.O., Universidad de La Laguna, 38271 La Laguna, Spain

\section{L.J. Hernández Paricio luis-javier.hernandez@unirioja.es}

Dpto. de Matemáticas y Computación, Universidad de La Rioja, 26004 Logroño, Spain

M. Marañón Grandes miguel.maranon@unirioja.es

Dpto. de Matemáticas y Computación, Universidad de La Rioja, 26004 Logroño, Spain

M.T. Rivas Rodríguez maria-teresa.rivas@unirioja.es

Dpto. de Matemáticas y Computación, Universidad de La Rioja, 26004 Logroño, Spain 